\title{
Design of a Novel Sensing Module for Machine Tool Vibration Monitoring by Integrating Multiple MEMS Accelerometers
}

\author{
Jui-Min Tsai ${ }^{\text {a, }}$, Kuo-Shen Chen ${ }^{\text {a }}$, Syuan-Wen Chen ${ }^{\mathrm{a}}$, Yun-Hui Liu ${ }^{\mathrm{b}}$, Da-Huei Lee ${ }^{\mathrm{c}}$ \\ ${ }^{a}$ Department of Mechanical Engineering, National Cheng-Kung University, \\ No.1 Daxue Rd., East Dist., Tainan City 701, Taiwan \\ ${ }^{b}$ Department of Mechanical Engineering, Southern Taiwan University of Science and Technology, \\ No.1 Nantai St., Yungkang Dist., Tainan 71005, Taiwan \\ ${ }^{c}$ Department of Electronic Engineering, Southern Taiwan University of Science and Technology, \\ No.1 Nantai St., Yungkang Dist., Tainan 71005, Taiwan \\ *Corresponding Author: rachelhahayaya@gmail.com
}

\begin{abstract}
Machine tools play key roles in modern manufacturing industry. The quality of machined products is largely depended on the status of machines in various aspects such as vibration and appropriate vibration monitoring would be essential for both quality control and the safety and life assessment of machine tools. However, currently used piezoelectric or MEMS (microelectromechanical systems) based accelerometers all have their own sensitivity and bandwidth limitations and may not suitable for machine tool monitoring, where both high sensitivity and high bandwidth are required. In this work, a design to form a vibrating sensing module by integrating several MEMS accelerometers with different dynamic characteristics is proposed and investigated. By such an approach, a low cost accelerometer with superior dynamic behavior at any frequencies expected. This work presents the preliminary study toward this goal, including overall conceptual design, sensor calibration, amplification and signal conditioning circuits layout, and preliminary integration, testing, and demonstration on a CNC machine tool. The primary test results are promising and it is believed that the design would play important role in future machine monitoring tasks.
\end{abstract}

Keywords: MEMS accelerometer, design of integration, machine tool monitoring.

\section{Introduction}

With the expeditious development of technology, the claim of Industry 4.0 has been advocated in order to accelerate productivity and decrease production cost. Cyberphysical systems, which establish the connection with mechanism and its users on the internet and making users understand the circumstance of it in time, are the key components for realizing the above goals in Industry 4.0 $\operatorname{era}^{(1)}$. One of the key task is on monitoring the health condition of machine tools for the purpose of status monitoring and system early warning. Among all possible physical signals, machine vibration is particular meaningful for condition monitoring of machine tools, where the sources of vibration are quite complicate, such as gear meshing, bearing damage, shaft rotation imbalance, and chattering during cutting. Vibrations caused by these sources have different time and frequency characteristics and usually distributed in a range around $10 \mathrm{kHz}$. Traditionally, piezoelectric accelerometers have been used for measuring vibrations in this domain ${ }^{(2)}$. However, a single piezoelectric accelerometer may not capable to faithfully measure the vibration of high speed machine tools due to its tradeoff between sensitivity and bandwidth. It is also expensive and the available space constrained by hiring multiple piezoelectric accelerometers.

Recently, with the advanced in MEMS (microelectromechanical systems) technology, MEMS accelerometers become an alternative choice in vibration measurement and they have been widely used in automobile and mobile communications. Recently, 2-axis MEMS accelerometers are also used to monitor rotating machine, such as motor ${ }^{(3)}$. Previously, the design of 3-axes MEMS 
accelerometers have also been applied on robot $\operatorname{arm}^{(4)}$. However, their performances are usually inferior and any single MEMS accelerometer also suffer from the same sensitivity-bandwidth tradeoff as having seen in piezoelectric accelerometers.

Despite of the above concerns, MEMS accelerometers are still attractive due to its nature of low cost and miniaturization design. In addition, individual MEMS accelerometers have exhibited acceptable performance in specific applications. Technically, it is possible to integrate several MEMS accelerometers together to resolve the tradeoff without changing the overall dimension significantly. As a result, the development of such a module of integrated MEMS accelerometers is a non-trivial task.

Motivated by the above needs, the overall goal is therefore to develop such a design of integrated MEMS accelerometers for monitoring the health condition of machine tool. Technically, there are four steps to achieve the final goal. First, several MEMS accelerometers must be integrated for optimizing the overall performance. Second, additional circuitry should be layout for filtering, rectifying, and amplifying the signals from accelerometers. Third, appropriated data reduction schemes should be coded in the associate firmware for effectively data processing to achieve meaningful index. Finally, these processed data could be uploaded to cloud for performing final status monitoring, early warning, and other tasks involving human decision depending on the applications and interested signal frequency using big data or $\mathrm{AI}$ technologies in the future.

In this work, we mainly present the efforts on MEMS accelerometers integration and circuitry layout for signal processing as the first step toward the final goal. The entire investigation flow is shown in Fig.1. Three types of MEMS accelerometers with different dynamic characteristics are chosen for performing this study.

Meanwhile, for ensuring the better orthogonality in measured acceleration, a small cubic adapter is used and all MEMS accelerometers as well as the primary circuitry board are attached on the adapter. The initial demonstration of the integrated MEMS accelerometer will be conducted by integrating three ADXL001 single axis accelerometers on this adapter to form a single 3-axis accelerometer and used for measuring the vibration of a 5 -axis $\mathrm{CNC}$ miller.

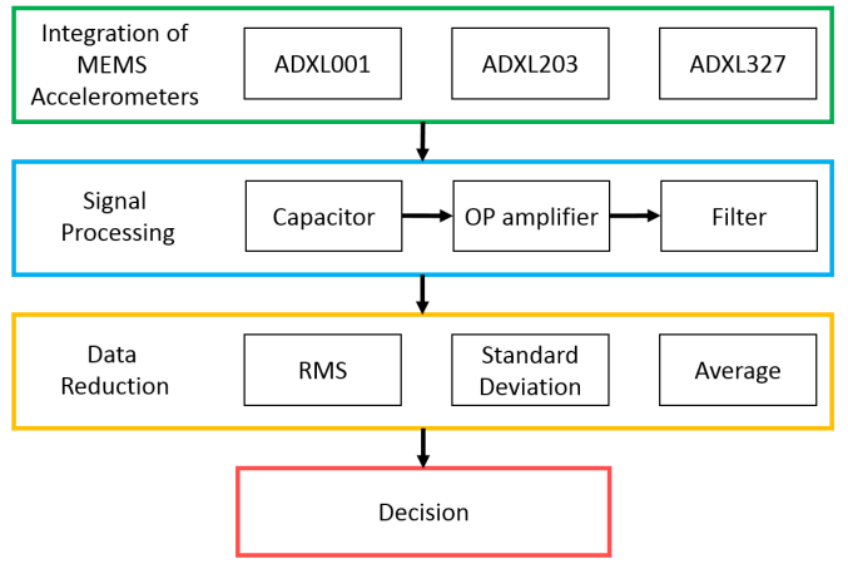

Fig.1 Overall investigation flow for designing of the integrated MEMS accelerometers

\section{Analysis and Design}

\subsection{Conceptual design}

The research flow chart is shown in Fig.2 and the main target is to establish a module by integrating three types of MEMS accelerometers and apply it for status monitoring of machine tools. As addressed earlier, before achieving the final objective, several tasks should be finished. First, it is important to choose appropriate accelerometers. Second, calibrating the MEMS accelerometers to ensure its dynamic characteristics for design. Finally, designing appropriated circuits for processing signals from MEMS accelerometers. More details are highlighted below.

\subsection{Selection of MEMS accelerometers}

There are three types of MEMS accelerometer selected owing to their different dynamic characteristics. For detecting high frequency vibration, the Analog Device ADXL001 single axis accelerometer is chosen because of its wide bandwidth (up to $22 \mathrm{kHz}$ ). However, its sensitivity is quite low (only $25 \mathrm{mV} / \mathrm{g}$ ). Meanwhile, for detecting vibration in medium frequency range, the dual axis ADXL203 is chosen. The bandwidth of this sensor can be up to $5.5 \mathrm{kHz}$ and the sensitivity dual $(1000 \mathrm{mV} / \mathrm{g})$ is much better. Finally, for handling low frequency vibrations, the triaxial ADXL327 is chosen. It has a relative low bandwidth $(1.6 \mathrm{kHz})$ and a moderated sensitivity $(420 \mathrm{mV} / \mathrm{g})$. We classify those types of sensor to three categories, high, 
middle, and low bandwidths, as shown as Fig.3.

\subsection{Calibration of MEMS accelerometers}

The above-mentioned technical data provided by vendor can only serve as references and essential calibration must be performed for system design. Here a piezoelectric accelerometer ( $\mathrm{PCB}, 354 \mathrm{C} 03)$ is used as the benchmark comparison. According to the method of back to back calibration $^{(5)}$, the measuring direction of MEMS accelerometer should be placed perpendicular to the piezoelectric accelerometer, shown in Fig.4. The sensitivity of MEMS accelerometer is calculated by

$$
S_{M E M S}=S_{\text {Piezo }} \times \frac{V_{M E M S}}{V_{\text {Piezo }}}
$$

Where $\mathrm{V}_{\text {MEMS }}$ and $\mathrm{V}_{\text {Piezo }}$ are the acceleration reported from both the MEMS and the piezoelectric accelerometers. In addition, for altering the bandwidth of MEMS accelerometers, the capacitance of the shunted capacitor should also be changed.

\subsection{Circuit layout}

The output of MEMS accelerometers consists of a DCbiased AC signal. To enhance the sensitivity, the AC signal should be further amplified. To achieve this, the DC bias should be removed in order to achieve a larger amplification before saturation. Both DC bias elimination and signal amplification can be achieved by incorporating OP-AMP based circuitry. Meanwhile, essential analog filters may also need to be implemented for suppressing undesirable noises. The schematic and layout of the circuit is shown in Fig.5.

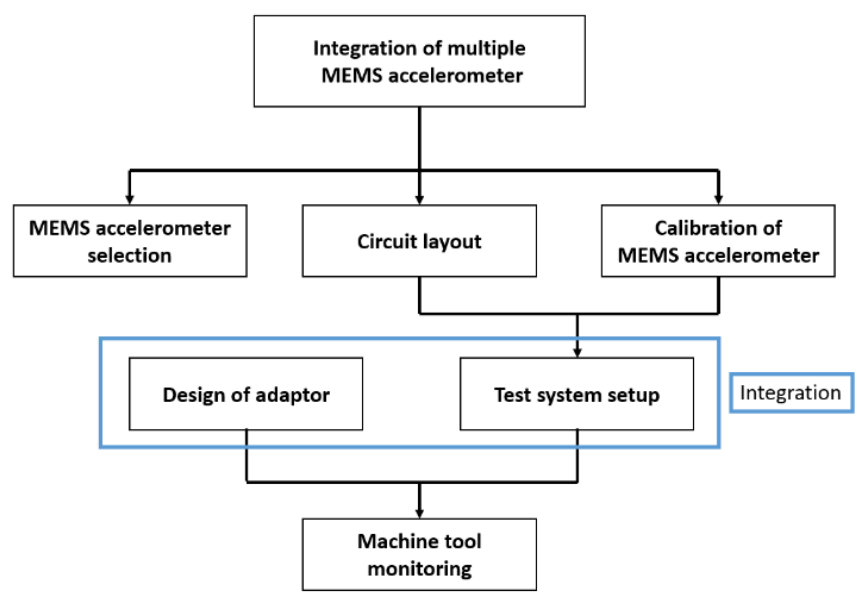

Fig.2 Investigation flow of the integrated accelerometer module

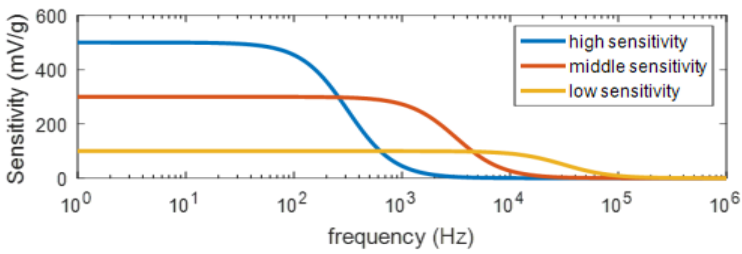

Fig.3 Schematic plot to indicate the frequency behavior of MEMS accelerometers

(a)

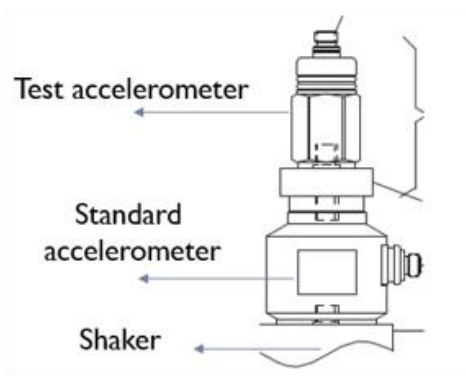

(b)

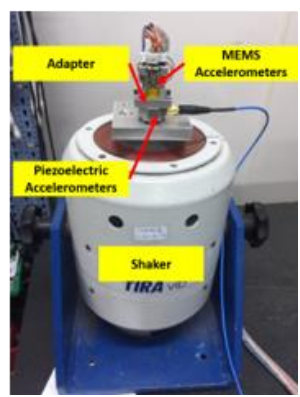

Fig.4 (a) The schematic diagram of back to back calibration $^{(6)}(b)$ The setup of the calibration experiment (a)

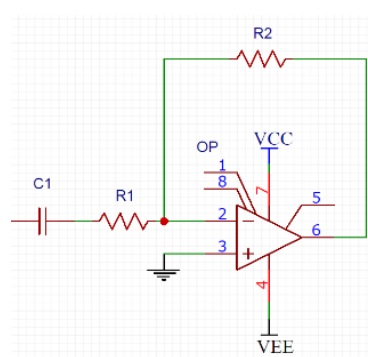

(b)

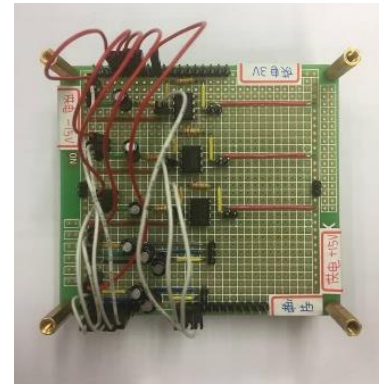

Fig.5 (a) The schematic diagram of the circuit (b) The circuit layout

\section{Testing system setup}

\subsection{Circuit bandwidth testing}

To ensure circuit would not influence the signal, it is necessary to measure the bandwidth of circuit. The sine signal provided by a function generator (Tektronix, AFG3021) from $20 \mathrm{~Hz}$ to $10 \mathrm{kHz}$ with an amplitude of $0.5 \mathrm{~V}$ as the input. As shown in Fig.6, the output signal is amplified by a ratio of 10 and this amplification ratio is essentially constant for signal frequency less than $10 \mathrm{kHz}$, which is higher than the bandwidth of the module. As a result, this implies that the circuit dynamics is sufficient fast and can be treated as a pure amplifier in the module. 


\subsection{MEMS accelerometers bandwidth test}

The accelerometer testing system is shown in Fig.7. MEMS accelerometers such as ADXL001 are fixed on the adapter, while the piezoelectric accelerometer (PCB, 354C03) served as the reference standard is also screwed in the same reference surface, and its characteristics is shown in Table.1. The shaker (TIRA, TV51120) outputs a sweep signal, which is measured by both accelerometers, and the sensor signals are acquired by a spectrum analyzer (B\&K type $3560 \mathrm{C}$ ) for computing their frequency characteristics. The results are shown in Fig.8(a). The sensitivity of ADXL001 is $25 \mathrm{mV} / \mathrm{g}$ when it is supplied by a $5 \mathrm{~V}$ power input, and its bandwidth is about $4700 \mathrm{~Hz}$, which is much smaller than the vendor's data. We believe that this is due to the limitation of our shaker, which has a maximal bandwidth of $7 \mathrm{kHz}$. Meanwhile, ADXL203 is measured by the same setup. As shown in Fig. 8 (b)(c), the. sensitivity and bandwidth are $1000 \mathrm{mV} / \mathrm{g}$ and $2900 \mathrm{~Hz}$ in $\mathrm{X}$-axis, $2600 \mathrm{~Hz}$ in $\mathrm{Y}$-axis under a supplied power of $5 \mathrm{~V}$. Finally, as shown in Fig. 8(d)(e)(f), the sensitivity and bandwidth of ADXL327 is around $370 \mathrm{mV} / \mathrm{g}$ and $1600 \mathrm{~Hz}$ in $\mathrm{x}$ axis, $1300 \mathrm{~Hz}$ in $\mathrm{y}$ axis, and $900 \mathrm{~Hz}$ in $\mathrm{z}$ axis respectively, based on a $3.3 \mathrm{~V}$ power input.

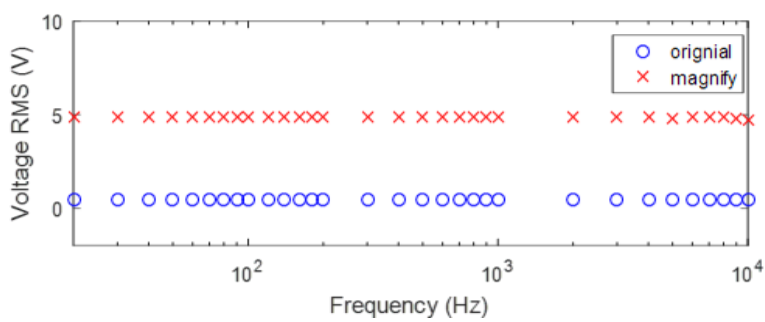

Fig. 6 The frequency response of the amplification circuit

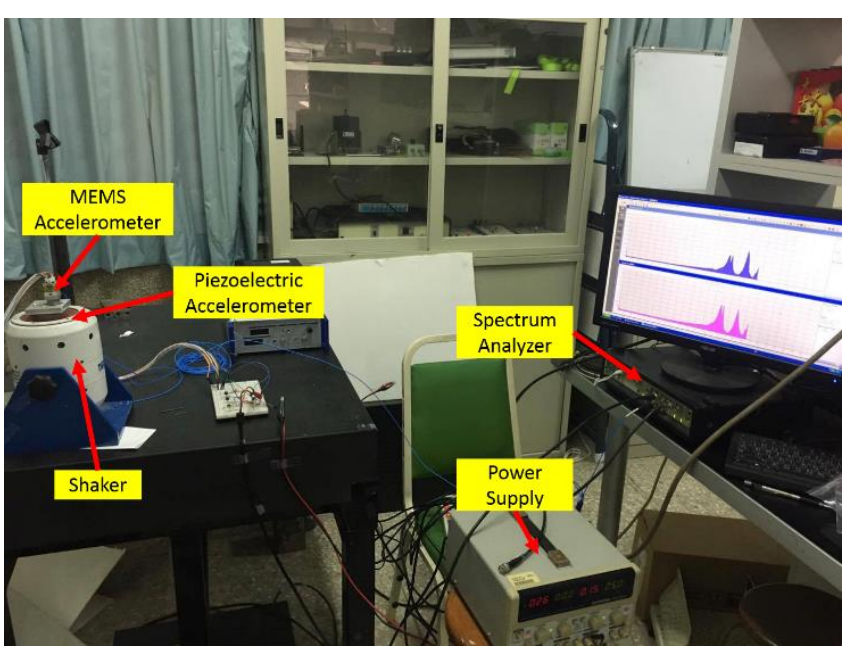

Fig.7 The accelerometer calibration system (a)
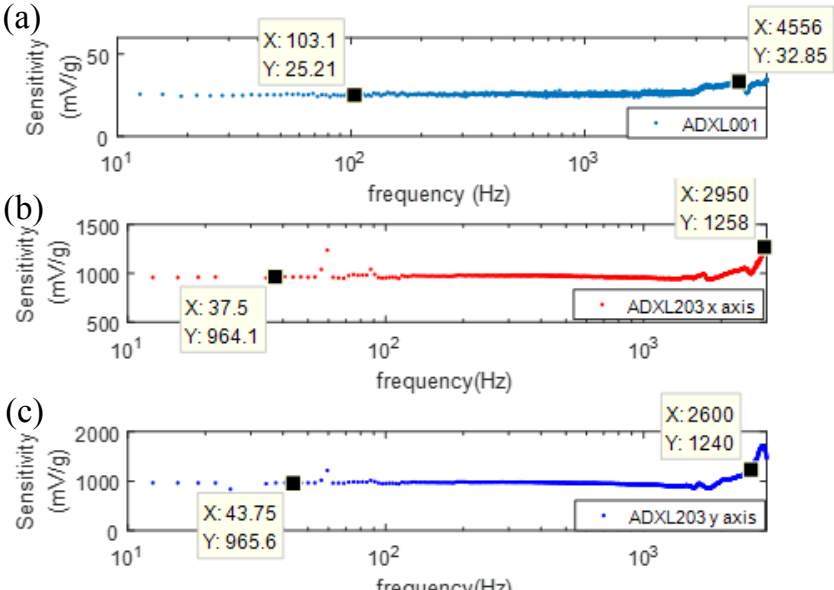

(d)

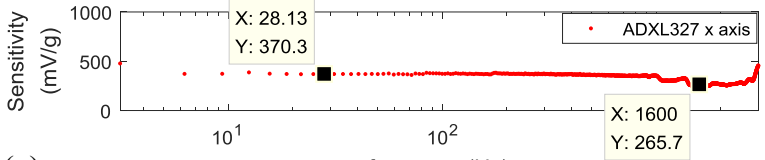

(e)

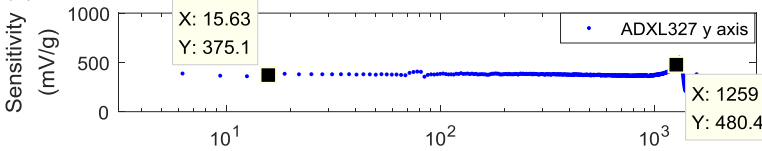

(f)

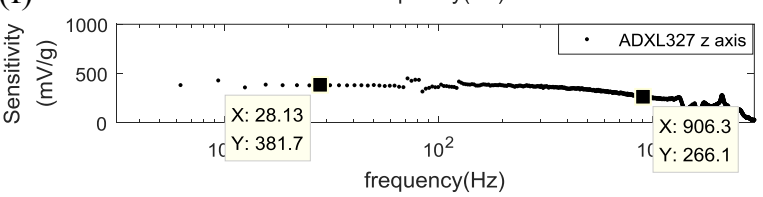

Fig.8 The frequency response of (a) ADXL001(b) the $\mathrm{x}$ axis of ADXL203 (c) the y axis of ADXL203 (d) the x axis of ADXL327 (e) the $y$ axis of ADXL327 (f) the $z$ axis of

ADXL327

Table 1. The characteristics of PCB 354C03 and Kistler 8763BB accelerometers

\begin{tabular}{|c|c|c|c|}
\hline \multicolumn{1}{|c|}{ Property } & Direction & Sensitivity $(\mathrm{mV} / \mathrm{g})$ & Bandwidth $(\mathrm{Hz})$ \\
\hline \multirow{2}{*}{ M.del } & $\mathrm{X}$ & 97.6 & \\
\hline & $\mathrm{Y}$ & 101.4 & 6000 \\
\hline \multirow{3}{*}{ Kistler 8763BB } & $\mathrm{Z}$ & 103.7 & \\
& $\mathrm{X}$ & 95.1 & 10000 \\
& $\mathrm{Y}$ & 110.4 & \\
\hline & $\mathrm{Z}$ & 108.2 & \\
\hline
\end{tabular}

\section{System Integration}

\subsection{Design of integrating MEMS accelerometers}

An adaptor is designed as the platform for adapting all MEMS accelerometers and their associate electronics. Since ADXL001 is single axis sensor, three orthogonal surface should be used to place three of them. On the other hand, 
ADXL203 and ADXL327 can be placed in any surface, depending on the usage. The remaining surfaces can be used for mounting necessary electronics and line adapters. The design is shown in Fig.9. The printed circuit board of MEMS accelerometers are reduced to $1 \mathrm{~cm} \times 1 \mathrm{~cm}$ for minimizing the size of module. There are three ADXL001, one ADXL203 and one ADXL327 on the adaptor in total. In addition, the signals are output to a circuit board on the top of the adaptor first to serve as a buffered connector for preventing the signals from disturbing by vibration, like a connector. Finally, a cable is used to transmit the signals to the amplifiers.

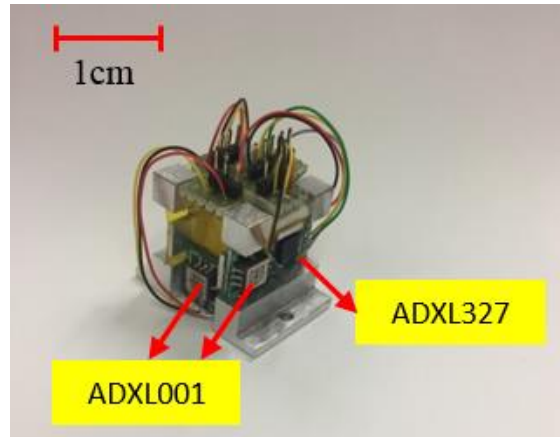

Fig.9 The prototype of the integrated module

\section{Demonstration test}

\subsection{Machine tool vibration measurement using ADXL001}

In this section, the MEMS accelerometer, ADXL001, is used for measure the vibration of the spindle of a CNC miller as the first step toward the fault diagnosis. In order to mimic an unhealthy spindle on the machine tool, such as the misalignment of cutters, a special cutter is designed to introduce unbalance intentionally. The entire test system is shown in Fig.10. Both a MEMS accelerometer (ADXL001) and a piezoelectric accelerometer (Kistler, 8763BB) are mounted on the spindle. A data acquisition system (NI 9215) to acquire signals with a sampling rate of $20000 \mathrm{~Hz}$. The spindle runs with rotational speed from 1200 to $12000 \mathrm{rpm}$ and the resulted vibration signals are shown in Fig.11 and Fig. 12 for both accelerometers, indicating that the vibration amplitude increases with rotating speed. However, since the sensitivity of ADXL001 is low and it requires amplification by a factor of 10. After amplification, the resulted vibration spectrums are similar. As shown in Fig.13, both accelerometers can capture the major frequency sparks at a $1200 \mathrm{rpm}$ speed. On the other hand, for a speed of $9600 \mathrm{rpm}$, the ADXL001 captures the rotation speed very well but fails to capture a low frequency vibration seen by the Kistler accelerometer possibly due to its inferior performance at extremely low frequency and this confirms the importance of using an additional low frequency MEMS accelerometer to achieve a successful measurement.

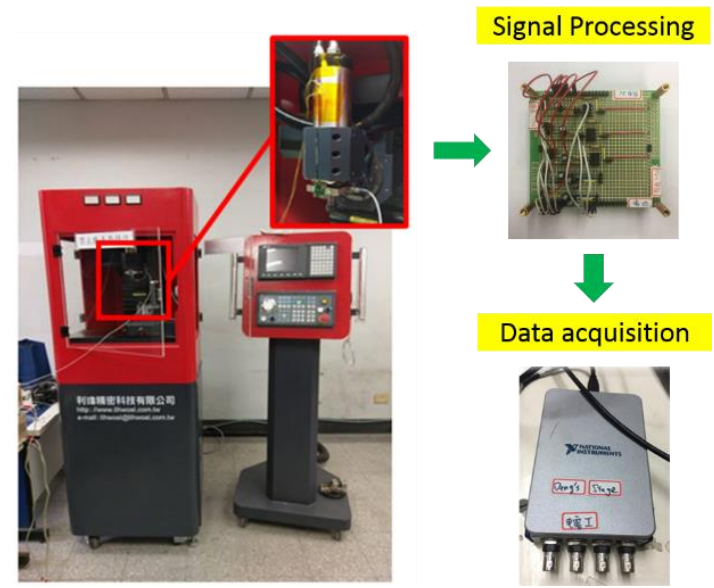

Fig.10 The experimental system for machine vibration monitoring
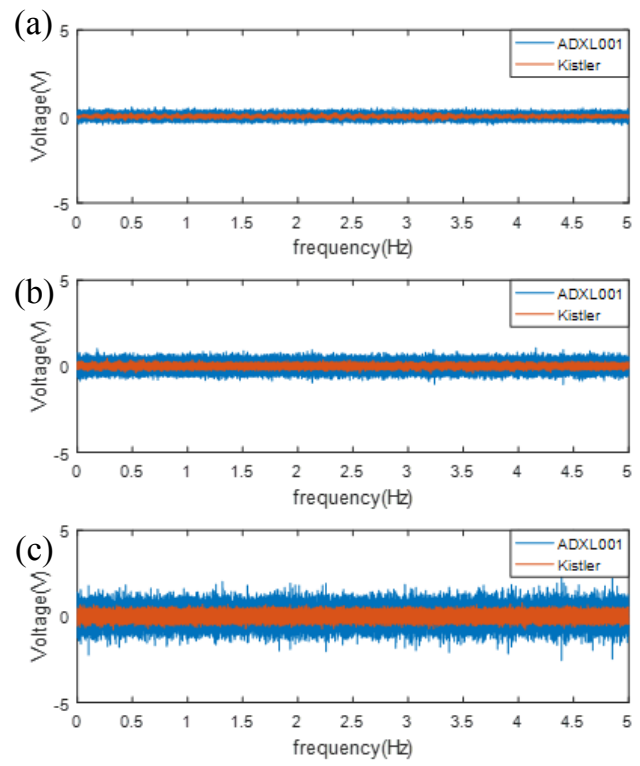

Fig.11 The measured unbalance accelerations in (a) 1200

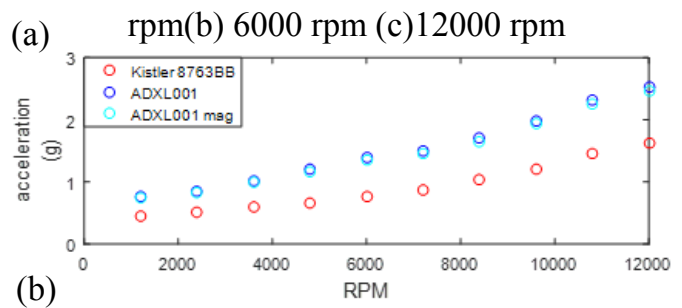

(b)

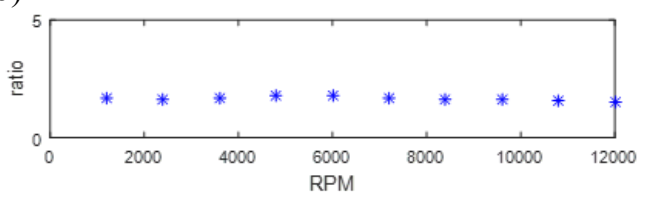

Fig.12 (a) RMS of the time acceleration signal (b) The sensitivity ratio between ADXL001 and Kistler 8763BB 
(a)
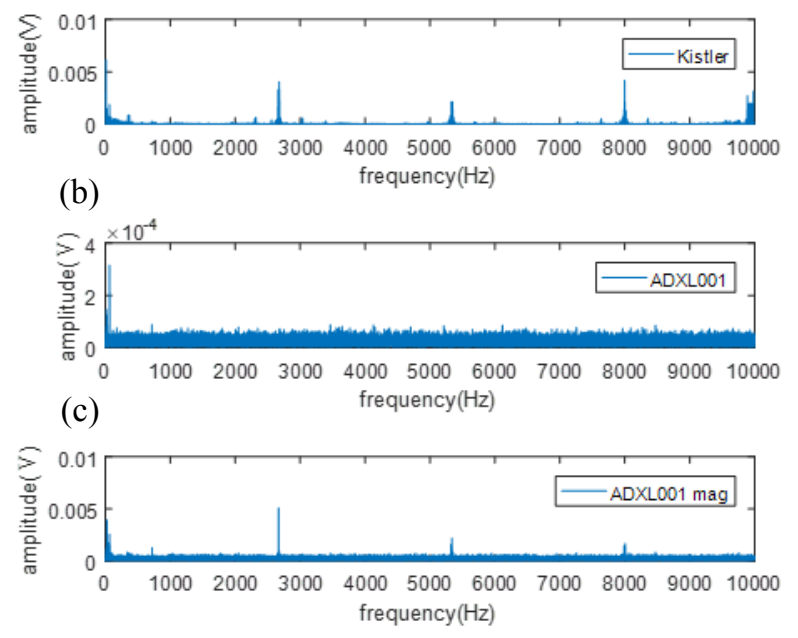

Fig.13 The frequency response of the measured acceleration in $1200 \mathrm{rpm}$ (a) Kistler 8763BB (b) ADXL001 (c) Magnified signal of ADXL001

(a)

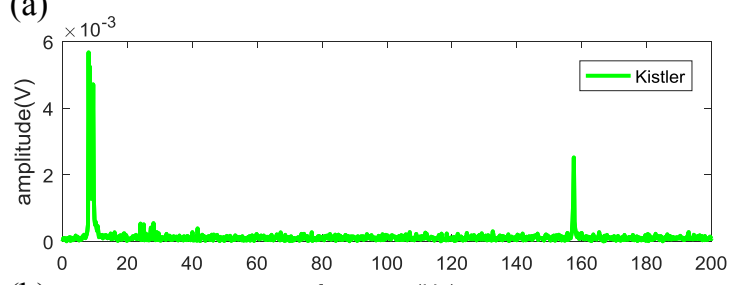

(b)

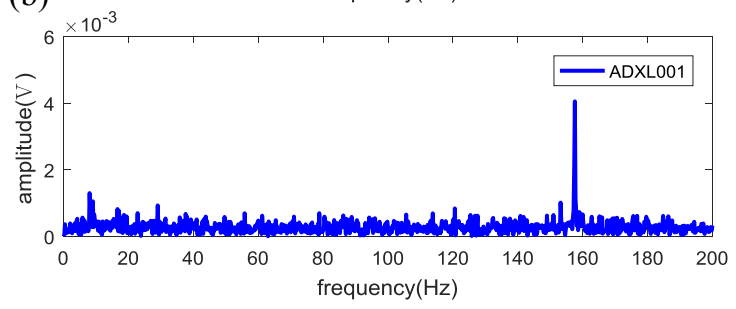

Fig.14 The frequency response in $9600 \mathrm{rpm}$ (a) Kistler 8763BB (b)Magnified signal of ADXL001

\subsection{Machine tool vibration measurement using the integrated module}

The spindle vibration measurement using ADXL001 proves the feasibility of using MEMS accelerometers for characterizing the vibration characteristics of machine tools. The next issue to be investigated is to apply the integrated module for monitoring the vibration of the same CNC miller during milling of copper. A straight path cutting is used in this work with a spindle rotating speed between $1000-8000$ rpm and a feed rate and a cutting depth of $300 \mathrm{~mm} / \mathrm{min}$ and $0.1 \mathrm{~mm}$, respectively. Typical spindle vibration histories during cutting are shown in Fig.15. Here the module contains only 3 ADXL001 accelerometers. Both the Kistler 8763BB and ADXL001 measure the three axes vibration of the spindle. Take $6000 \mathrm{rpm}$ for example, the time signal of both ADXL001 and Kistler can clearly observe the vibration generated from milling process but the responses from ADXL001 seem to be a little bit noisy. This fact can also be observed in the measured power spectrum shown in Fig.16, where the Kistler clearly identifying the major components but the module contains considerable noisy components. It is believed that improper shielding in current data transmission cable from the sensor module to the circuitry unit is the major contributor. In the future, the noise isolation would be a major challenge to be overcome. Nevertheless, the major frequency components can still be found and this proves the feasibility of the designed module.

(a)

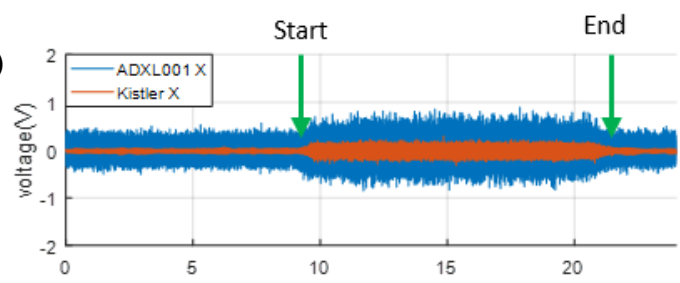

(b)
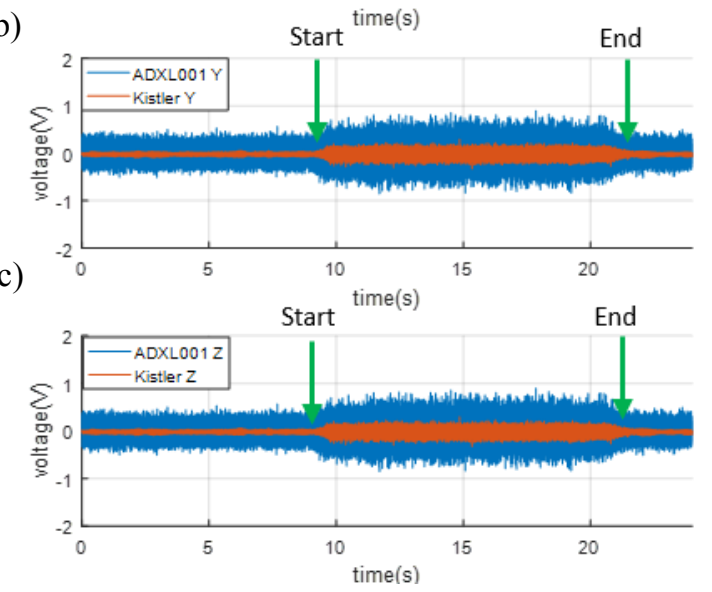

Fig.15 Sensor output histories in 6000rpm (a) $x$ axis (b) y axis (c) z axis

(a)

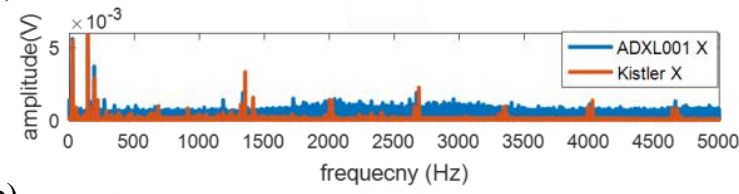

(b)

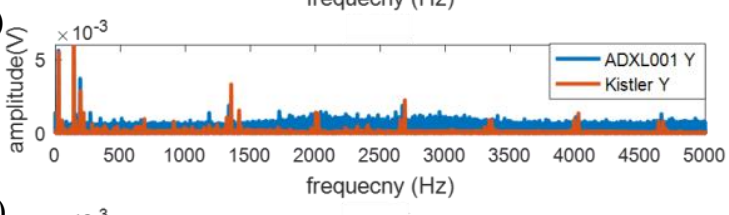

(c)

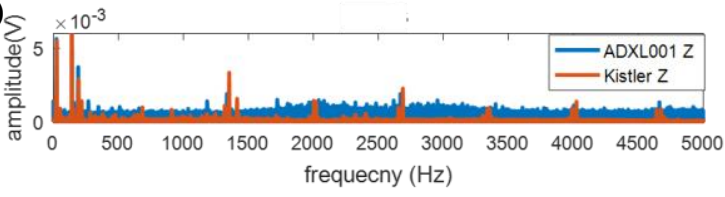

Fig.16 Frequency response of the measured acceleration in 6000rpm (a) $x$ axis (b) y axis (c) z axis 


\section{Discussion}

This work mainly presents the concept and initial progress of designing a vibration measurement module for machine tools by integrating multiple MEMS accelerometers. Currently, the dynamic characteristics of all selected MEMS accelerometers have been obtained. The chosen MEMS accelerometers could possibly achieve a very good balance in low frequency sensing sensitivity and high frequency measuring bandwidth.

Meanwhile, based on the test results on the CNC miller, it is concluded that the performance of individual ADXL001 is actually better than the corresponding Kistler piezoelectric accelerometer. However, in our integrated module, the performance is actually not as expected. We believe that this is due to the additional noise generation generated by improper shielding and layout of our signal transmission and module packaging. In the future, this issue needs to be solved by a thoughtful shielding design. Nevertheless, the feasibility of the design is still proven by the test results.

In the near future, in addition to conduct the effort of noise reduction, other accelerometers such as ADXL 203 and ADXL 327 would be integrated into the module. More sophisticated design in circuit layout and transmission will also be implemented for providing improved quality of signals and better dynamic characteristics are expected. Finally, after finishing such a fully integrated module, the next goal is to further miniaturize the sensor module itself. This should be achieved by further reducing the size of the adapter and a more thoughtful layout of MEMS accelerometer chips and the supplementary circuits, as well as adapting the state of the art circuit layout. With such efforts, we believe that such a practical low-cost vibration measurement module could be realized soon and make substantial contributions in machine tool status monitoring tasks.

\section{Conclusions}

In this article, a design to form a vibrating sensing module by integrating several MEMS accelerometers with different dynamic characteristics is proposed and investigated. By such an approach, a low cost accelerometer with superior dynamic behavior at any frequencies is expected. This work presents the preliminary study toward this goal. The primary test results on the dynamic performance characterization indicated that those MEMS accelerometers are potentially capable for measuring vibrations of machine tools. The in-situ on spindle also indicated that the performance of MEMS accelerometer could potentially compete with that of piezoelectric ones. Meanwhile, the test results also indicated that the current performance of the integrated module is limited by the sensor noises and should be further improved. In the future, full scale integration of multiple types of MEMS accelerometers will be conducted and the scale would be further reduced for fitting the space constraints in machine tool applications.

\section{Acknowledgment}

This research is supported by the Ministry of Science and Technology in Taiwan, ROC under the contact numbers 105-2221-E-006-100-MY and 106-2221-E-218-014.

\section{References}

(1) J. Lee, H-A Kao, and S. Yang: "Service innovation and smart analytics for industry 4.0 and big data environment", Procedia CIRP, Vol. 16, pp. 3-8, 2014.

(2) J.K. Sinha, K. Elbhbah : "A future possibility of vibration based condition monitoring of rotating machines", Mechanical Systems and Signal Processing, Vol. 34, pp. 231-240, 2013.

(3) S. B. Chaudhury, M. Sengupta, K. Mukherjee : "Vibration Monitoring of Rotating Machines Using MEMS Accelerometer", International Journal of Scientific Engineering and Research, Vol. 2, pp. 5-11, 2014.

(4) A. A. Jaber and R. Bicker : "Design of a Wireless Sensor Node for Vibration Monitoring of Industrial Machinery," International Journal of Electrical and Computer Engineering, Vol. 6, pp. 639-653, 2016.

(5) R. Darula and A. Sjöström : " Construction and InHouse Calibration of Mems-Based Vibration Transducers", in Engineering Acoustics, LTH, Lund University ,(TVBA-3131), 2016.

(6) Dytran, schematic diagram of back to back calibration, http://www.dytran.com/assets/PDF/Back-toBack\%20Accelerometer\%20Calibration.pdf 